\title{
Hypoglycemia In The Emergency, But What Awaits Us For Cardiac Risks In The Future?
}

\author{
Onur Akhan ${ }^{1}$ and Isa Ardahanlı ${ }^{2}$ \\ ${ }^{1}$ Affiliation not available \\ ${ }^{2}$ Bilecik Training and Research Hospital
}

January 7, 2021

\begin{abstract}
Introduction: Several homeostatic changes like an increase in sympathoadrenal response and oxidative stress occur in hypoglycemia. As a result of these findings, an increase in inflammation and pre-atherogenic factors is observed and these changes may lead to endothelial dysfunction. Aim: Our study aims to reveal possible cardiac risks (systolic-diastolic functions and endothelial dysfunctions) in patients who have applied to the emergency department with hypoglycemia. Methods: This crosssectional, case-control study included 46 hypoglycemia patients who admitted to the emergency with symptoms compatible with hypoglycemia and diagnosed with hypoglycemia and 30 healthy volunteers. All patients were evaluated with baseline echocardiography, tissue-doppler imaging(carotid and brachial artery). Also, the fasting blood tests of the patients referred to the internal medicine department were examined. Results: There were no differences between the groups regarding age, weight, body mass index, and systolic blood pressure. Total cholesterol, LDL, HDL, Vitamin B12, TSH, and fasting blood glucose levels were similar in the groups' blood tests (all p values $>0.05$ ). We observed a statistically significant decrease in diastolic dysfunction parameters: $\mathrm{E} / \mathrm{A}$ and $\mathrm{E} / \mathrm{e}$ ' ratios (respectively, $\mathrm{p}=0.020$ and 0.026 ). It was shown that insulin resistance was influential in forming these considerable differences. The patient group observed that the carotid intima-media thickness was more remarkable $(\mathrm{p}=0.001)$, and the brachial flow-mediated dilatation value was smaller $(\mathrm{p}=0.003)$, giving an idea about endothelial functions.
\end{abstract}

\section{Introduction}

Hypoglycemia, also known as low blood sugar, is a condition that can be symptomatic and not very rare. A significant part of it is seen from organic reasons that disrupt the physiological response required for blood sugar. It can cause generally mild symptoms like palpitation, tremor, anxiety, sweating, hunger, dizziness, presyncope, confusion, and fatigue, but severe cerebrovascular symptoms such as seizures and loss of consciousness in acute and chronic processes can also be seen $(1,2)$. Symptoms compatible with hypoglycemia, low plasma glucose concentration, and disappearance of symptoms when plasma glucose level rises are requirements in the primary diagnosis of hypoglycemia, also known as the Whipple triad $(3,4,5)$.

In the pathophysiology of hypoglycemia, it has been shown that increased insulin sensitivity, exaggerated insulin response to insulin resistance or glucagon-related peptide increase, increased adrenergic sensitivity, increased epinephrine levels, and impaired glucagon regulation may occur. Emotional stress, anxiety, depression, as well as after heavy alcohol and high carbohydrate diets. Hypoglycemia is also common in diabetes and especially with drugs used in treatment $(6,7,8)$.

The primary threshold value in the diagnosis of hypoglycemia is accepted as $70 \mathrm{mg} / \mathrm{dl}$ and can also be used in hypoglycemia classification. In addition to the blood sugar being below $70 \mathrm{mg} / \mathrm{dl}$, the symptoms of hypoglycemia and the elimination of these symptoms only with the help of another person can be defined as severe, hypoglycemia symptoms and regression of these symptoms after blood glucose-raising agents 
defined as symptomatic hypoglycemia and the situation had no symptoms can be defined as asymptomatic hypoglycemia. Although the blood sugar is above $70 \mathrm{mg} / \mathrm{dl}$, hypoglycemia-like symptoms can be seen in the condition described as pseudo-hypoglycemia $(1,9)$.

Hypoglycemia induces a stress response that leads to sympathoadrenal activation and the release of hormones such as glucagon, epinephrine, cortisol, and growth hormone $(10,11)$. Hemodynamic changes such as increased heart rate, myocardial contractility, and systolic blood pressure may be observed to glucose formation from the liver and glucose support to the brain $(12,13,14)$. Also, blood viscosity may increase with an increase in platelet cells and increased aggregation and coagulation. Inflammation, leucocytosis, lipid peroxidation, oxidative stress are also increased with hypoglycemia and cause endothelial dysfunction. Disruption of endothelium-related vasodilation and even vascular tone deterioration causes an increased risk of atherosclerosis $(15,16,17,18)$.

When the relevant literature was searched, it was seen that the effects of hypoglycemia on endothelial factors, especially in diabetic patients, were investigated $(16,17,18)$. Besides, in a study conducted in rats, the relationship of recurrent hypoglycemia with carotid intima thickness was shown (19). However, there is no study in the literature evaluating diastolic functions in healthy individuals presenting hypoglycemia attacks. Also, studies on endothelial functions in these individuals are generally conducted at the molecular level to investigate situations that may cause possible future risks $(16,17,20)$.

In the light of these pieces of information, our study aimed to reveal possible cardiac risks by evaluating the cardiac effects (especially in terms of systolic and diastolic functions) and endothelial functions in the presence of echocardiographic findings and peripheral doppler imaging in patients admitted to the emergency department with a diagnosis of hypoglycemia.

\section{Materials and Methods}

Our study is a cross-sectional, case-control study. The local ethical committee approved this study. Patients between the ages of 18 and 50 admitted to the emergency department with hypoglycemia symptoms and whose blood glucose was 70 and below were included in the study. The fasting routine blood samples were investigated by examining these patients' internal medicine-endocrinology department records after their emergency room administration. Hypoglycemia patients whose cardiology application was recommended mainly due to palpitation symptoms, dizziness, and presyncope, were evaluated in terms of essential echocardiographic evaluation, tissue doppler, and flow-mediated dilatation.

Patients diagnosed with diabetes mellitus, hypertension, hyperlipidemia, coronary artery disease, congestive heart failure, peripheral artery disease, moderate to severe heart valve disease, cardiomyopathies, thyroid dysfunction, chronic obstructive pulmonary disease, malignancy, rheumatological disease, active infection, kidney failure, liver disease, obesity (body mass index $>30$ ), drug usage(including beta-blockers), and patients with heavy alcohol intake and smoking were excluded from the study. A total of 46 patients were included in the survey by excluding patients with low echogenicity who were not suitable for imaging. Thirty healthy individuals who were not diagnosed with hypoglycemia before were included in the study as the control group.

The HOMA-IR is being used extensively for estimates of beta-cell function and insulin resistance and calculated with the formula '(Fasting insulin in $\mathrm{mIU} / \mathrm{L} *$ fasting blood glucose in $\mathrm{mg} / \mathrm{dL}$ ) / 405'. A HOMA-IR value of 2.5 and above was accepted as insulin resistance (21).

The groups with and without insulin resistance were determined based on the HOMA-IR scores of the patient group, and it was evaluated whether the differences with the control group were related to insulin resistance.

\section{Image Recordings and Analysis}

Standard echocardiographic measurements; EF with modified Simpson method, tissue doppler measurements, and cardiac valve evaluations performed with transthoracic echocardiography (EPIQ 7 echocardiography device (Philips, Amsterdam, Netherlands)) by two cardiologists. 2015 ASE (American Society of 
Echocardiography) guideline used as a reference for standard echocardiographic measurements (22). In echocardiographic tissue doppler evaluations, the myocardial performance index(MPI) is an index that incorporates both systolic and diastolic time intervals in expressing global systolic and diastolic ventricular function, also known as Tei Index, was also evaluated. MPI calculated with the formula '[Isovolumetric contraction time (IVCT) +Isovolumetric relaxation time(IVRT)]/Ejection Time(ET)', for both left ventricle lateral and septal parts (23).

Carotid systolic and diastolic diameters, intima-media thickness (IMT), and brachial flow-mediated dilatation performed with duplex ultrasound (Toshiba Sonolayer SSA 270 A equipped with a $7.5 \mathrm{Mhz}$ linear array transducer, Toshiba Medical Systems, Japan). The carotid IMT measurements were performed in the distal $1 \mathrm{~cm}$ of each common carotid artery's far wall and plaque-free segments. The mean carotid IMT value was derived from the measurements made in both carotid arteries' locations (24). Two cardiologists perform all examinations and measurements.

All ultrasonic examinations were performed in a semi-dark room with continuous ECG. The brachial artery was evaluated by keeping the patient's left arm still, in a horizontal plane, and a linear array transducer. Brachial artery diameters were assessed at the diastolic phase, approximately $2 \mathrm{~cm}$ above the elbow, in the longitudinal plane. Basal measurements were evaluated after 15 minutes of rest in the fasting state. The appropriate sphygmomanometer cuff located in the patient's upper arm was then inflated to the level of 200 $\mathrm{mmHg}$ and waited for 4 minutes. Continuous recordings were made until 2 minutes after cuff deflation. The maximum rate of change was calculated considering the maximum measured diameter and resting brachial artery and the basal measurement. Peak flow-mediated dilatation of brachial artery (bFMD) measured in a percentage $(\%)$ and calculated with the formula '[(maximum diameter - basal diameter)/basal diameter ${ }^{*} 100$ ' $(25,26)$.

\section{Statistical Methods}

SPSS 26.0 and Modeler 18.0 (IBM Corporation, Armonk, New York, United States) programs were used to analyze variables. Univariate data's suitability to normal distribution was evaluated with the Shapiro-Wilk Francia test, while variance homogeneity was assessed with the Levene test. Independent-Samples T-test was used together with Bootstrap results. In contrast, the Mann-Whitney U test was used together with Monte Carlo to compare two independent groups according to quantitative data. One-Way Anova test, one of the parametric methods, was used to compare multiple separate groups according to quantitative data. The Tukey HSD test was used for post hoc analysis. Kruskal-Wallis H Test, one of the nonparametric tests, was used with Monte Carlo simulation technique results, and Dunn's test was used for Post Hoc analysis. In comparison of categorical variables, Pearson Chi-Square Exact results were analyzed, while the Fisher-Freeman-Holton test was tested with the Monte Carlo Simulation technique.

Logistic Regression, Support Vector Machine, Random Forest, K-nearest Neighbor Algorithm, Simple (Native) Bayes Classification, and Neural Network (Multilayer Perceptron-Radial Basis) were used to find and predict the variable with the highest significance in the patient and control groups. Neural Network (Multilayer Perceptron) analysis, which is the most successful model among these methods, was used. Gradient descent was used for optimization algorithm, Hyperbolic tangent as hidden layer activation function, Softmax as output Layer activation function were used. The Mini-Batch method was used for the training data selection, and a $70 \%$ Trial set was set as a 30\% Testing set. Quantitative variables are mean \pm SD (standard deviation) in tables. Moreover, Median (Percentile 25\% / Percentile 75\%), while categorical variables were shown as n (\%). Variables were analyzed at a $95 \%$ confidence level, and a p-value of less than 0.05 was considered significant.

\section{Results:}

There was no significant difference between the patient and control groups regarding age, gender, height, weight, BMI, and systolic blood pressure in our study. Although diastolic blood pressure was significantly lower in the control group, the values were within the normal reference range $(p=0,003)$. Total cholesterol, LDL, HDL, Vitamin B12, TSH, and fasting blood glucose levels were similar in the groups' blood tests. Fast- 
ing insulin levels and HOMA-IR were significantly higher in the patient group ( $p$ values $<0.001$ ). Although significant differences were found in triglyceride and liver function test levels between the patient and control groups, the values determined were within the normal reference range. The demographic characteristics and blood analysis of the patient and control groups are shown in Table 1.

When the groups' echocardiographic characteristics were examined, heart rate and left ventricular ejection fractions were similar ( $p$ values $>0,05$ ). No significant difference was observed in the patients' tissue doppler evaluation in terms of E, A waves, lateral and septal e, a, s waves, IVCT, IVRT, ET, and RVSm (Right ventricular peak systolic myocardial velocity). E/A and E/e' ratios, which are essential parameters for diastolic dysfunction, were significantly lower in the patient group (respectively, $p$ values are 0.020 and 0.026), but the deceleration time was similar in both groups. When the myocardial performance index was evaluated, no significant difference was observed for the lateral MPI value. At the same time, the septal MPI value was found to be significantly higher than the control group.

There was no significant difference between the two groups in aortic systolic and diastolic diameters and carotid artery systolic and diastolic diameters. In peripheral doppler evaluation with the linear array transducer, carotid intima-media thicknesses were significantly higher in the patient group than in the control group (Median values 0.58 and 0.51 , respectively, $p=0.001$ ). Brachial flow-mediated dilatation was lower in the patient group than the control $(6.59+-0.52,6.96+-0.49, p=0.003)$. Echocardiographic findings are shown in Table 2 .

Table 3 shows the characteristics of the patient group with and without insulin resistance and the control group. The relationship of these differences with insulin resistance and its statistical significance are summarized in Table 4 and detailed in the discussion section.

\section{Discussion}

Hypoglycemia causes several homeostatic changes to balance falling blood sugar (11). Changes in hemodynamic response can be seen in healthy individuals, especially in diabetic patients, with sympathoadrenal response after hypoglycemia. Also, an increase in inflammatory and pro-atherogenic markers was detected as a result of oxidative stress. Combining these factors revealed a rise in blood viscosity, endothelial dysfunction, and acceleration of the atherosclerotic process. It has been shown that insulin resistance can accelerate cardiac morbidity and mortality caused by hypoglycemia by worsening these processes, especially in diabetic patients $(16,17,18,20,21)$.

Due to the lack of a detailed study showing the impact of hypoglycemia on diastolic functions and the conditions mentioned above, it was aimed to reveal possible cardiac risks in patients who applied to the emergency department with hypoglycemia and who were referred to cardiology with complaints of palpitations, dizziness, and presyncope. For this purpose, brachial flow-mediated dilatation and carotid intima-media thickness were examined to show endothelial dysfunction, and essential echocardiographic and tissue doppler evaluation was performed to reveal systolic and diastolic effects.

When looking in the literature regarding systolic and diastolic parameters, an increase in myocardial contractility secondary to transient sympathoadrenal response during acute hypoglycemia after insulin administration in healthy subjects was detected by multiple-gated radionuclide ventriculography (27). A study found that hypoglycemia worsened survival in diabetic patients with systolic heart failure (28). In large-scale ADVANCE, ORIGIN, NICE-SUGAR studies conducted in patients with type 2 diabetes, the relationship of hypoglycemia with outcomes of heart failure, coronary artery disease, and peripheral artery disease has been demonstrated $(29,30,31)$. From these studies, it can be inferred that systolic dysfunction outcomes due to hypoglycemia may be observed. Fasting blood glucose was shown to be one of the markers in the Young Finns study conducted in a population aged between 34 and 49 years to show the determinants of diastolic dysfunctions. However, in this study, high blood glucose, the diagnostic criterion for metabolic syndrome, was mentioned (32).

In our study, in terms of systolic functions, there was no difference(mean LVEF: 65.85+-3.75 (patient), 
$64.57+-5.55$ (control); $p=0.285$ ). The deceleration time did not differ between the groups in terms of diastolic parameters $(p=0.058)$. When the $\mathrm{E} / \mathrm{A}$ ratio was examined, it was observed that it was lower in the patient group(Patient (1.33+-0.34), control $(1.52+-0.34), p=0.020)$. It was shown that insulin resistance was influential in the formation of this significant difference $(p($ Homa- $I R<2.5$ vs. control $)=0.249, p(H o m a-$ $I R$ [?]2.5 vs. control $)=0.013)$. Similarly, when the $\mathrm{E} / \mathrm{e}$ ' ratios were examined, it was significantly lower in the patient group (Patient (median 4,53), control (median 5.10), $p=0.026$ ). Besides, this rate was considerably more down in the patient group with insulin resistance than the patient group without insulin resistance

$(p($ Homa-IR<2.5 vs. Homa-IR/?]2.5 $)=0,035, p($ Homa-IR<2.5 vs. control $)=0$,238, p(Homa-IR/?]2.5 vs. control) $=0,002)$.

Although the diastolic early filling time (E wave duration) was lower in the patient group than the control group, this difference was not statistically significant $(p=0.052)$. However, the $\mathrm{E}$ wave duration with insulin resistance was statistically more down in the group than the control group (68.60+-19.24 (Homa-IR/?]2.5), $83.50+-16.35$ (Control)); $p=0.020)$. It was revealed that the main reason for the significant differences in $\mathrm{E} / \mathrm{A}$ and $\mathrm{E} / \mathrm{e}$ ' ratios was the difference in $\mathrm{E}$ wave duration, also with the considerable effect of insulin resistance.

Studies conducted with insulin resistance have shown that insulin resistance may lead to subclinical myocardial and vascular irregularities, such as decreased global longitudinal strain values and arterial stiffness in individuals without significant coronary artery disease. Insulin resistance, directly and indirectly, causes adverse effects in the vascular area. Endothelial dysfunction and disruption of the vasomotor response to the pro-inflammatory, pro-coagulant endothelium can be listed among these effects (33). In another study, insulin resistance has been shown to increase the likelihood of ischemia-related cardiac dysfunction (34). It can be thought that the stated effects of insulin resistance may be among the reasons for the differences in diastolic parameters we found in our study.

Prediabetic patients without previous coronary artery disease have been shown to have higher mpi scores than healthy populations, which has been associated with possible subclinical systolic and diastolic dysfunction (35). In our study, the septal mpi score (Tei index) was found to be significantly higher in patients with hypoglycemia, and no significant difference was found in the lateral mpi score ( $p=0.045$ and $p=0.474$; respectively)

Similar effects of recurrent hypoglycemia have been demonstrated in animal experiments with mice, as demonstrated as increased carotid intima-media thickness due to hyperglycemia in persons with metabolic syndrome $(19,36)$. Interestingly, an increase in the circulating catecholamine levels causes a decrease in the peripheral arterial resistance through $\beta$-2 receptor-mediated vasodilation. In the acute phase of hypoglycemia in healthy people, vascular elasticity increases. Additionally, increased catecholamine levels cause a decrease in peripheral arterial resistance with beta-2 receptor-associated vasodilation. However, hypoglycemia episodes (especially in diabetic patients) have been reported in previous studies to cause arterial stiffness. It has been shown that hematological and hemodynamic changes during hypoglycemia may play a role in this situation $(12,14,37)$.

In our study, the patient group observed that the carotid intima-media thickness was more remarkable, and the brachial flow-mediated dilatation value was smaller, which is an indicator of endothelial dysfunction. It was revealed that findings related to carotid intima-media thickness was marked by insulin resistance $(p($ Homa $-I R<2.5$ vs. control $)=0.027, p($ Homa $-I R[?] 2.5$ vs. control $)<0.001)$. Still, in terms of brachial flowmediated dilatation, when the groups with and without insulin resistance were compared with the control group, the significance levels were found to be $\operatorname{similar}(p($ Homa-IR<2.5 vs. control) $=0.027, p$ (Homa-IR[?]2.5 vs. control $)=0.028)$. We can state that these findings are compatible with the pathophysiological process in related studies $(12,14,21,34,37)$.

Considering the study population's age group, the findings determined predominantly in terms of diastolic functions (also with endothelial functions) may be predictive for other cardiac pathologies. It was also revealed that the increase in insulin resistance might affect the distinction in diastolic function, septal 
isovolumetric relaxation time, and carotid intima-media thickness parameters found among the study groups.

We can state that our study will contribute to the literature in the light of echocardiographic findings and peripheral examinations after further evaluations of patients admitted to the emergency department with hypoglycemia. These findings may guide possible future studies that will be conducted with many patients' participation and in which cardiac involvement can be demonstrated in more detail (such as strain echocardiography, 3D echocardiography).

\section{Limitations}

A limited number of patients could be included in the study. Only the evaluation of patients referred to the cardiology unit and all patients' failure to come due to the Covid-19 pandemic process; patients included in the study may not reflect the targeted population.

Considering the study groups' basal characteristics, the differences in diastolic blood pressure and triglyceride levels might affect the investigation results, even if the relevant parameters are found in the standard reference range. Similarly, vitamin B12 levels were within the normal reference range in the patient group with and without insulin resistance; it was significantly lower in patients with insulin resistance than those without insulin resistance and control. This difference may have affected the results.

\section{Conclusion}

As a message, we can say that hypoglycemia may affect diastolic functions in addition to endothelial dysfunction. Therefore, even young individuals without any chronic diseases may need follow-up in terms of possible risks.

\section{Acknowledgments}

The authors declare no conflict of interest in the study, and no financial support is received. We would like to thank our hospital's emergency department and internal diseases department for their helpfulness in conducting our study.

\section{Author contributions}

Concept/design: Onur Akhan, Data analysis/interpretation: Onur Akhan, Isa Ardahanli, Drafting article: Onur Akhan, Critical revision of article: Onur Akhan, Approval of article: Onur Akhan, Isa Ardahanli, Data collection: Onur Akhan, Isa Ardahanli

\section{References}

1. Seaquist ER, Anderson J, Childs B, et al. hypoglycemia and diabetes: a report of a workgroup of the american diabetes association and the endocrine society. Diabetes Care. 2013;36:1384-1395.

2. DeRosa MA \& Cryer PE. Hypoglycemia and the sympathoadrenal system: neurogenic symptoms are largely the result of sympathetic neural, rather than adrenomedullary, activation. American journal of physiology. Endocrinology and metabolism 2004287 E32-41

3. Whipple AO. The surgical therapy of hyperinsulinismus. J Int Chir 1938 3 237-276.

4. Whipple AO. Islet cell tumors of the pancreas. Canadian Medical Association journal 195266 334-342.

5. Kittah N.E., Vella A. MANAGEMENT OF ENDOCRINE DISEASE: Pathogenesis and management of hypoglycemia Eur J Endocrinol 2017 Jul;177(1):R37-R47.

6. Iqbal A, Heller S Managing hypoglycaemia Best Pract Res Clin Endocrinol Metab 2016 Jun;30(3):41330 .

7. The Diabetes Control and Complications Trial Research Group. The effect of intensive insulin treatment of diabetes on the development and progression of long term complications in insulin-dependent diabetes mellitus. N Engl J Med 1993; 329:977-986.

8. Cariou B, Fontaine P, Eschwege E. Frequency and predictors of confirmed hypoglycemia in type 1 and insulin-treated type 2 diabetes mellitus patients in a real-life setting: results from the DIALOG study. Diabetes Metabolism. 2015;41(2):116-125. 
9. Cryer PE. Preventing hypoglycaemia: what is the appropriate glucose alert value? Diabetologia 2009; 52(1): 35-7.

10. Benson JW Jr, Johnson DG, Palmer JP. Glucagon and catecholamine secretion during hypoglycemia in normal and diabetic man. J Clin Endocrinol Metab. 1977 Mar;44(3):459-464.

11. Cryer PE \& Gerich JE. Glucose counterregulation, hypoglycemia, and intensive insulin therapy in diabetes mellitus. N Engl J Med 1985313 232-241.

12. Frier BM, Schernthaner G, Heller SR. Hypoglycemia and cardiovascular risks. Diabetes Care. 2011;34(suppl 2):S132-S137.

13. Sanon V.P., Sanon S., Kanakia R. Et al. Hypoglycemia from a cardiologist's perspective. Clin Cardiol. 2014 Aug;37(8):499-504. doi: 10.1002/clc.22288. Epub 2014 Jun 4.

14. Wright RJ, Frier BM. Vascular disease and diabetes: is hypoglycaemia an aggravating factor? Diabetes Metab Res Rev. 2008;24:353-363.

15. Singh P, Jain A, Kaur G. Impact of hypoglycemia and diabetes on CNS:correlation of mitochondrial oxidative stress with DNA damage. Mol Cell Biochem 2004;260:153-159

16. Gogitidze Joy N, Hedrington MS, Briscoe VJ, Tate DB, Ertl AC, Davis SN. Effects of acute hypoglycemia on inflammatory and pro-atherothrombotic biomarkers in individuals with type 1 diabetes and healthy individuals. Diabetes Care 2010;33:1529-1535

17. Wang J, Alexanian A, Ying R, et al. Acute exposure to low glucose rapidly induces endothelial dysfunction and mitochondrial oxidative stress: role for AMP kinase. Arterioscler Thromb Vasc Biol 2012;32:712-720

18. Joy NG, Tate DB, Younk LM, Davis SN. Effects of acute and antecedent hypoglycemia on endothelial function and markers of atherothrombotic balance in healthy humans. Diabetes 2015;64:2571-2580

19. Yasunari E., Mita T., Osonoi Y. Et al. Repetitive hypoglycemia increases circulating adrenaline level with resultant worsening of intimal thickening after vascular injury in male Goto-Kakizaki rat carotid artery 2014 Jun;155(6):2244-53.

20. Al-Qaissi A, Papageorgiou M, Deshmukh H, et al. Effects of acute insulin-induced hypoglycaemia on endothelial microparticles in adults with and without type 2 diabetes. Diabetes Obes Metab. 2019 Mar;21(3):533-540.

21. Matthews DR, Hosker JP, Rudenski AS, et al. Homeostasis model assessment: insulin resistance and beta-cell function from fasting plasma glucose and insulin concentrations in man. Diabetologia. 1985 Jul;28(7):412-9.

22. Lang RM, Badano LP et al. Recommendations for cardiac chamber quantification by echocardiography in adults: an update from the American Society of Echocardiography and the European Association of Cardiovascular Imaging. J Am Soc Echocardiogr (2015) 28(1):1-39.

23. Shingu Y, Amorim P, Nguyen TD, et al. Myocardial performance (Tei) index is normal in diastolic and systolic heart failure induced by pressure overload in rats, Eur J Echocardiogr, Volume 11, Issue 10, December 2010, Pages 829-833.

24. Stein JH, Korcarz CE, Hurst RT, et al. Use of carotid ultrasound to identify subclinical vascular disease and evaluate cardiovascular disease risk: a consensus statement from the American Society of Echocardiography Carotid Intima-Media Thickness Task Force. Endorsed by the Society for Vascular Medicine. American Society of Echocardiography Carotid Intima-Media Thickness Task Force. J Am Soc Echocardiogr. 2008 Feb; 21(2):93-111; quiz 189-90.

25. Corretti MC, Anderson TJ, Benjamin EJ, et al. Guidelines for the ultrasound assessment of endothelialdependent flow-mediated vasodilation of the brachial artery: A report of the International Brachial Artery Reactivity Task Force, J Am Coll Cardiol., Volume 39, Issue 2, 2002, Pages 257-265.

26. Bots ML, Westerink J, Rabelink TJ, et al. Assessment of flow-mediated vasodilatation (FMD) of the brachial artery: effects of technical aspects of the FMD measurement on the FMD response, European Heart Journal, Volume 26, Issue 4, February 2005, Pages 363-368.

27. Fisher BM, Gillen G, Dargie HJ, et al. The effects of insulin-induced hypoglycaemia on cardiovascular function in normal man: studies using radionuclide ventriculography. Diabetologia. 1987 Nov;30(11):841-5. 
28. Hsu PF, Sung SH, Cheng HM, et al. Association of clinical symptomatic hypoglycemia with cardiovascular events and total mortality in type 2 diabetes: a nationwide population-based study. Diabetes Care. 2013;36:894-900.

29. The ADVANCE Collaborative Group (George Inst for International Health, Sydney, NSW, Australia; et al) Intensive Blood Glucose Control and Vascular Outcomes in Patients with Type 2 Diabetes N Engl J Med 2008; 358:2560-2572

30. The ORIGIN Trial Investigators. Basal insulin and cardiovascular and other outcomes in dysglycemia. N Engl J Med 2012;367:319-28.

31. Finfer S, Heritier S; NICE Study Management Committee and SUGAR Study Executive Committee. The NICE-SUGAR (Normoglycaemia in Intensive Care Evaluation and Survival Using Glucose Algorithm Regulation) Study: statistical analysis plan. Crit Care Resusc. 2009 Mar;11(1):46-57.

32. Heiskanen JS, Ruohonen S, Rovio SP, et al. Determinants of left ventricular diastolic function-The Cardiovascular Risk in Young Finns Study. Echocardiography. 2019 May;36(5):854-861.

33. Novo G, Manno G, Russo R, et al. Impact of insulin resistance on cardiac and vascular function. Int J Cardiol. 2016 Oct 15;221:1095-9.

34. Jelenik T, Flögel U, Álvarez-Hernández E, et al. Insulin Resistance and Vulnerability to Cardiac Ischemia. Diabetes. 2018 Dec;67(12):2695-2702.

35. Askin L, Cetin M, Tasolar $\mathrm{H}$, et al. Left ventricular myocardial performance index in prediabetic patients without coronary artery disease. Echocardiography. 2018 Apr;35(4):445-449.

36. Sengul C, Cevik C, Ozveren O, et al. Echocardiographic epicardial fat thickness is associated with carotid intima-media thickness in patients with metabolic syndrome. Echocardiography. $2011 \mathrm{Sep} ; 28(8): 853-$ 8.

37. Sommerfield AJ, Wilkinson IB, Webb DJ, et al. Vessel Wall stiffness in type 1 diabetes and the central hemodynamic effects of acute hypoglycemia. Am J Physiol Endocrinol Metab. 2007;293:E1274-E1279. 